\title{
Automatic Source Attribution of Text: A Neural Networks Approach
}

\author{
Foaad Khosmood and Franz Kurfess, Ph.D. \\ Department of Computer Science \\ California Polytechnic State University \\ San Luis Obispo, CA 93407 \\ foaadk@yahoo.com, fkurfess@calpoly.edu
}

\begin{abstract}
Recent advances in automatic authorship attribution have been promising. Relatively new techniques such as $\mathbf{N}$-gram analysis have shown important improvements in accuracy [2]. Much of the work in this area does remain in the realm of statistics best suited for human assistance rather than autonomous attribution [6]. While there have been attempts at using neural networks in the area in the past, they have been extremely limited and problem-specific [7]. This paper addresses the latter points by demonstrating a practical and truly autonomous attribution process using neural networks. Furthermore, we use a word-frequency classification technique to demonstrate the feasibility of this process in particular and the applications of neural networks to textual analysis in general. Key Words: neural networks, computational linguistics, authorship attribution, source attribution.
\end{abstract}

\section{INTRODUCTION}

We define automatic source attribution as the ability for an autonomous process to determine the source of a previously unexamined piece of text. A software system designed to follow such a process would analyze a set of input corpora, and construct a neural network to engage in attribution. It would then train the network with the corpora; apply the sample texts and determine attribution. For our source recognition problem, our system constructs a 5 layer, 420 Million-connection neural network. It is able to correctly attribute sample texts, previously unexamined by the system. Specifically, we conduct three sets of experiments to test the ability of the system: broad categorization, narrow categorization and minimal-sample categorization.

An automatic source attribution system must be able to digest a set of text corpora with known sources in order to determine the source or literary originator of a new piece of writing. The word "automatic" is meant to emphasize the desired absence of human intervention in the attribution process. Most of the work in source or authorship attribution is currently done with heavy involvement of humans. Even many computerized or statistical methods serve merely as assistants to human decision makers [6] who still to some extent subjectively evaluate the writing. This is true of almost all the famous authorship attribution cases. For example some statistical methods were used in assisting human specialists in determining The Federalist Papers dispute (some claimed by both Hamilton and Madison) [7].

We build on previous experience to make ours a problemindependent autonomous system.

\section{SOURCE VERSUS AUTHORSHIP}

Many of the previous works within the field of computer science refer to this area as "authorship attribution."

For a variety of reasons, we believe "source attribution" is a more accurate description of our experiments. The works of different individuals can appear together as part of the same unit with the same style and linguistic distinction. Associated Press news stories for example may be written by several different individuals but they all adhere to the same established writing style and may report about the same subject or even the same incident. The Bible and technical manuals are also examples of distinctive "sources."

There are thus multiple factors that constitute "source." Two of the most important ones are originator and subject matter. It is important to note that each of these spheres of contribution have shifting scopes that depend on other sources they are being distinguished from. Originator, for example, could mean "Shakespeare," or "British author" or "English language author" depending on what else it's being compared to. Similarly subject could be relatively narrow such as "US Foreign Policy in Latin America 1999-2000" or broad like "Love" or "Life." 


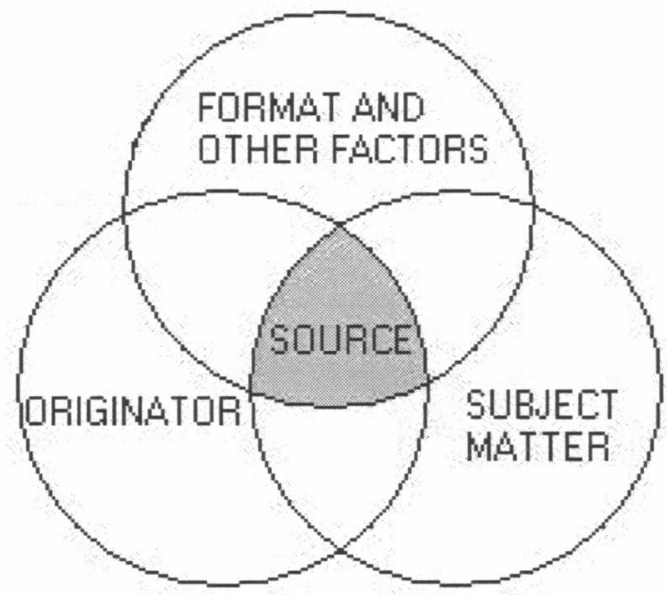

FIGURE 1, SOURCE

\section{ATTRIBUTION STRATEGY}

We use a common-word-frequency strategy to solve the attribution problem with neural networks. In this technique, a set of non-trivial words that are common to all corpora are derived from the set. These words make up the input layer of our neural network. The strength of each input signal is determined by the frequency of the word's occurrence in a particular corpus. The output layer consists of neurons each representing one source.

We design a 5 layer, back-propagation neural network with sigmoid activation functions and random initial weights. The size of the input and output layers are dynamically determined based on the problem set (i.e. the number of shared words and the number of corpora.) We fix the size of the middle three layers to 100,50 and 25 neurons respectively.

\section{SYSTEM DESIGN AND TOOLS}

The system accepts both a set of corpora and a set of "source-less" texts, each to be attributed to one of the corpora. The output layer consists of a small number of neurons each representing a source.

Our system is constructed on a Linux machine with $\mathrm{X}$ Windows support and PDP++ tool kit including CSS scripting support. Our program checks a certain directory for newly placed folders, each of which are named after one source and contain the corpus of training work for that source. The text or html files are properly parsed and analyzed for words. We used GNU Flex, BASH and a number of GNU tools to derive the set of in-common words. We use the PDP++ scripting features (CSS) to automatically specify a variable-sized neural net, train it and extract results.

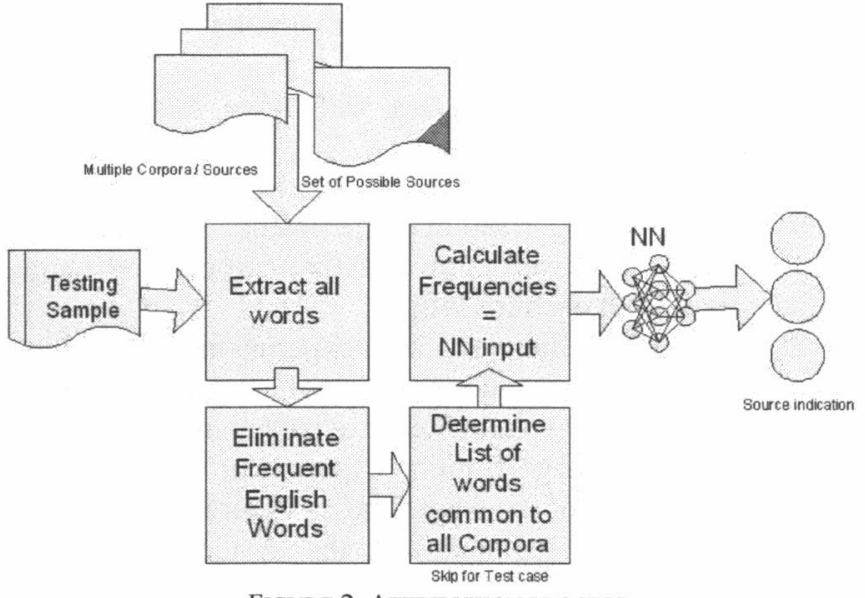

FIGURE 2, ATTRIBUTION PROCESS

Our word extractor ignores all lexemes with numerals and symbols, all XML style tags and a small set (roughly 40) of common English grammatical operators such as "and," and "or." Some symbols such as quotation mark, single quote and hyphen are removed and the surrounding two spaces are joined. All capital letters are converted to lower-case. After extraction, all repetitions are deleted and another 125 words most frequent in the English language, eliminated. The list of frequent words is provided by Kenneth Beare and is available for download on www.about.com.

In-common words are determined by examining all remaining words in all corpora. These words represent the universe of comparison among all the corpora. Each of the words has a corresponding input neuron in our network. For every word on the list, we determine the frequency in each source. Frequency is calculated by dividing the number of occurrences by the total number of words post-extraction but pre-elimination. The divisor thus will still not include counts of symbols and numbers but will likely contain counts of frequent English words, as well as repetitions.

Having derived the necessary information from the corpora, the system creates a CSS script that specifies the 5layer neural network. Normalized versions of the in-common, non-trivial, word frequencies are applied as input for training data sets. The corresponding corpus code constitutes the output layer. 


\section{EXPERIMENTS AND CORPORA}

We begin testing the system with three sets of source attribution experiments in mind.

1. Broad categorization experiment: We apply 5 diverse and well known textual corpora and sample texts from each for testing.

2. Declining sample size experiment: We apply successively smaller portions of a sample text to the trained system and observe accuracy in attribution.

3. Narrow categorization experiment: We consider categories of a single broad source as separate narrow sources and test the system's ability to distinguish sample texts between these new sources.

For the purposes of the first experiment we select the following five sources of text. Table 1 contains a description and size of each source.

\begin{tabular}{|l|l|l|}
\hline \multicolumn{4}{|c|}{ TABLE 1, SOURCE CORPORA } \\
\hline \multicolumn{1}{|l|}{ SOURCE } & \multicolumn{1}{l|}{$\begin{array}{l}\text { NUM. } \\
\text { WORDS }\end{array}$} \\
\hline Bible (B) & American Standard Bible & 31,102 \\
\hline Chomsky (C) & $\begin{array}{l}\text { Select Political Writings 1990- } \\
2004\end{array}$ & 4,823 \\
\hline Linux (L) & $\begin{array}{l}\text { Comments in Kernel Source } \\
\text { Code }\end{array}$ & 20,212 \\
\hline Poe (P) & All poems + "Fall of Usher" & 10,362 \\
\hline $\begin{array}{l}\text { Shakespeare } \\
\text { (S) }\end{array}$ & $\begin{array}{l}\text { Hamlet, Julius Caesar, } \\
\text { Othello and R+J. }\end{array}$ & 30,184 \\
\hline
\end{tabular}

\section{SAMPLE TEXTS AND RESUltS}

Our system derives 672 in-common, non-trivial words for this corpus set. Our neural network dimensions are thus $(672 \times 100 \times 50 \times 25 \times 5)$. The network generally converges within about 500 epochs. We use random initial weights for all connections. We measure convergence in our back propagation network by when the SSSE (Sum of Sum of Squared Errors) of the output neurons becomes insignificant. To calculate SSSE, our system subtracts each output neuron value from its intended training target (error). The error values of all the neurons in the output set are squared (SE) and added together (SSE). Finally the process is repeated for every distinct output set -which in this case is five- and those results are added together (SSSE.)

We applied the following sample texts (Table 2) for the broad categorization experiment. Results were positive in every case.

The confidence value is 100 times the output level of the correct output neuron. The neural output value ranges from 0 to 1 and is inversely related to SSE.
TABLE 2, BROAD CATEGORIZATION RESUlTS

\begin{tabular}{|c|c|c|c|c|}
\hline S\# & DESCRIPTION & SRC & WRDS & $\begin{array}{l}\text { CONFI- } \\
\text { DENCE }\end{array}$ \\
\hline 1 & Bible - entire corpus & $\mathrm{B}$ & 31,102 & $99.0591 \%$ \\
\hline 2 & $\begin{array}{l}\text { Chomsky - entire } \\
\text { corpus }\end{array}$ & C & 4,823 & 98.6381 \\
\hline 3 & Linux - entire corpus & $\mathrm{L}$ & 20,212 & 99.1525 \\
\hline 4 & Poe - entire corpus & $\mathrm{P}$ & 10,362 & 99.0206 \\
\hline 5 & $\begin{array}{l}\text { Shakespeare - entire } \\
\text { corpus }\end{array}$ & $\mathrm{S}$ & 30,184 & 98.9395 \\
\hline 6 & $\begin{array}{l}200 \text { line subset of Bible } \\
\text { corpus }\end{array}$ & B & 2,718 & 93.0880 \\
\hline 7 & $\begin{array}{l}1019 \text { line subset of } \\
\text { Linux corpus }\end{array}$ & $\mathrm{L}$ & 2,624 & 91.4564 \\
\hline 8 & $\begin{array}{l}260 \text { line subset of Poe } \\
\text { corpus }\end{array}$ & $\mathrm{P}$ & 1,193 & 99.2502 \\
\hline 9 & $\begin{array}{l}600 \text { line subset of } \\
\text { Shakespeare corpus }\end{array}$ & $\mathrm{S}$ & 1,567 & 98.2209 \\
\hline 10 & $\begin{array}{l}500 \text { lines of King James } \\
\text { Bible }\end{array}$ & B & 2,389 & 98.0366 \\
\hline 11 & $\begin{array}{l}\text { excerpt from } \\
\text { "Hegemony," Chom '03 }\end{array}$ & $\mathrm{C}$ & 942 & 98.2122 \\
\hline 12 & Interview in 2004 & $\mathrm{C}$ & 3,204 & 95.1804 \\
\hline 13 & Interview in 1991 & C & 8,016 & 87.3992 \\
\hline 14 & BLOG entries 2003-4 & $\mathrm{C}$ & 3,651 & 97.2593 \\
\hline 15 & $\begin{array}{l}1000 \text { line Ethernet } \\
\text { comments }\end{array}$ & $\mathrm{L}$ & 4,093 & 96.4314 \\
\hline 16 & VFS documentation & $\mathrm{L}$ & 2,084 & 97.0880 \\
\hline 17 & $\begin{array}{l}\text { Tell-Tale Heart and } \\
\text { Cask of Amontillado }\end{array}$ & $\mathrm{P}$ & 2,537 & 99.2060 \\
\hline 18 & King Lear & $\mathrm{S}$ & 1,764 & 98.9652 \\
\hline 19 & Mark:5 (New Am. Bible) & $\mathrm{B}$ & 722 & 72.3709 \\
\hline
\end{tabular}

Samples 1 through 5 are the actual source corpora. The high confidence values of these results stems from the low SSSE value and confirm the convergence of the neural network [1]. Samples 6 through 9 are small, random, contiguous subsets of the corpora and thus are known to the system. These results demonstrate our algorithm's relative independence from sample text size in broad categorization. The vocabulary usage pattern being recognized by the neural net is being exhibited in samples which are about an order of magnitude smaller than their respective corpora proper. But the system's confidence in attributing the subsets is not necessarily as high as for the corpus. Sample size will be explored further below in our declining sample size experiment.

Samples 10 through 19 have not been utilized in the training of the network and are thus true tests of our broad categorization experiment. The lowest performing piece is the last one from the New American Standard Bible which is attributed with $72 \%$ confidence. Two factors are most likely responsible for this score. The first is size. The NASB sample has the lowest word-count of all the samples testes. While we explore declining sample size with Chomsky corpus below, we must keep in mind that the Bible is a broader and less homogeneous corpus than Chomsky. Secondly, the NASB represents a significant and deliberate linguistic deviation 
from both the American Standard Bible and the King James Bible. The NASB translators strived for literalism with particular emphasis on original idioms and phraseology in original languages. One commentator has described it as "more Greek, less English" [9].

\section{DECLINING SAMPLE SIZE EXPERIMENT}

Using the same neural network that produced the above results, we applied successively smaller portions of Noam Chomsky's political writing to the system. The piece is the first chapter from Chomsky's 2004 book titled "Hegemony or Survival: America's Quest for Global Dominance" and is available for free online. The entire chapter contains 942 words. We apply the entire chapter (which is attributed with high confidence) and 6 other subsets ranging from 942 to 118 words. Each chapter subset is produced by simply removing a paragraph from the end of the previous subset. The results are noted in table 3 . They are remarkable in their accuracy of attribution, despite unusually small sizes. Even a sample of 118 words was correctly attributed to the Chomsky corpus with high confidence.

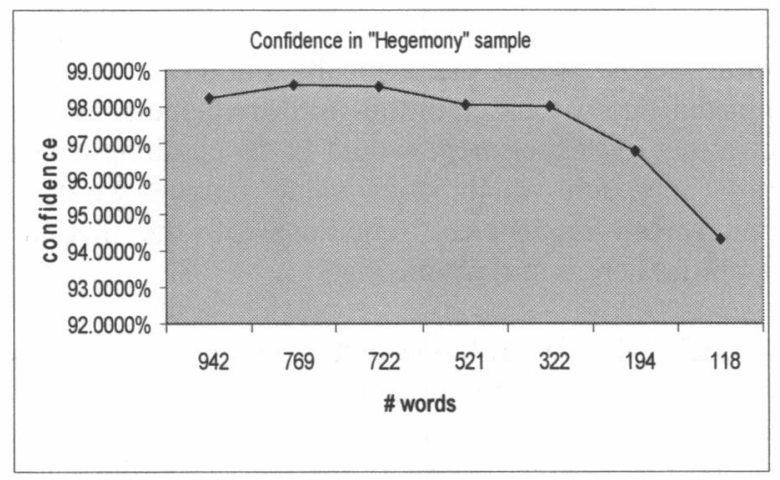

FIGURE 3, DECLINING SAMPLE SIZE EXPERIMENT

\section{NARROW CATEGORIZATION EXPERIMENT}

Our narrow categorization experiment concentrates on Shakespeare entirely. We utilize our automated process to construct a new neural network and train it with a new set of corpora consisting of Shakespeare's comedies, tragedies and sonnets. The system should be able to attribute sample texts to each category of Shakespearian writing [3].

TABLE 3. NARROW CATEGORIZATION SOURCES

\begin{tabular}{|l|l|l|}
\hline \multicolumn{2}{|c|}{ SOURCE } & \multicolumn{2}{|c|}{$\begin{array}{l}\text { NuM. } \\
\text { WORDS }\end{array}$} \\
\hline $\begin{array}{l}\text { Shakespeare's } \\
\text { comedies (C) }\end{array}$ & $\begin{array}{l}\text { Merchant of Venice, } \\
\text { Midsummer Night's Dream, } \\
\text { Taming of the Shrew, } \\
\text { Twelfth Night }\end{array}$ & \\
\hline $\begin{array}{l}\text { Shakespeare's } \\
\text { sonnets (S) }\end{array}$ & Sonnets 100 through 150 & 4,331 \\
\hline $\begin{array}{l}\text { Shakespeare's } \\
\text { tragedies (T) }\end{array}$ & $\begin{array}{l}\text { Hamlet, Julius Caesar, } \\
\text { Othello, Romeo and Juliet }\end{array}$ & 70,409 \\
\hline
\end{tabular}

Our process determined 962 common, non-trivial words in the three sources of comedies, tragedies and sonnets. A 5 layer, $360,750,000$ connection $(962 \times 100 \times 50 \times 25 \times 3)$, backpropagation network was created. As in the first two experiments, the sigmoid activation function and random initial weights were used. No special learning curve or acceleration variables were used and all neurons had identical thresholds. Once the training data were applied the network converged in about 150 epochs on average.

Since all three corpora are originated from Shakespeare and the writing styles are similar, the challenge becomes more significant for the neural network. We emphasize that the presence of key words, i.e. words inherently associated with one corpus, is not helpful in our process. "Romeo" for example is associated with a tragedy, but the word will not be part of our neural net since it is not shared among comedy, tragedy and sonnet corpora.

Table 4 contains results of the narrow categorization problem.

TABLE 4, NARROW CATEGORIZATION RESULTS

\begin{tabular}{|c|c|c|c|c|}
\hline S\# & DESCRIPTION & SRC & WRDS & CONF. \\
\hline 1 & $\begin{array}{l}\text { All's Well that } \\
\text { Ends Well }\end{array}$ & C & 403 & $99.5769 \%$ \\
\hline 2 & $\begin{array}{l}\text { Comedy of } \\
\text { Errors }\end{array}$ & C & 1256 & 99.4556 \\
\hline 3 & $\begin{array}{l}\text { Measure for } \\
\text { Measure }\end{array}$ & C & 1125 & $(98.6647)^{\star}$ \\
\hline 4 & $\begin{array}{l}\text { Much Ado } \\
\text { About Nothing }\end{array}$ & $\mathrm{C}$ & 317 & $(89.6729)^{*}$ \\
\hline 5 & As You Like It & $\mathrm{C}$ & 137 & 77.6646 \\
\hline 6 & $\begin{array}{l}\text { Antony and } \\
\text { Cleopatra }\end{array}$ & $\mathrm{T}$ & 1448 & $(89.0783)^{\star}$ \\
\hline 7 & King Lear & $\mathrm{T}$ & 1835 & $(98.1022)^{\star}$ \\
\hline 8 & Macbeth & $\mathrm{T}$ & 495 & 98.5415 \\
\hline 9 & Coriolanus & $\mathrm{T}$ & 921 & 97.9012 \\
\hline 10 & $\begin{array}{l}\text { Titus } \\
\text { Andronicus }\end{array}$ & $\mathrm{T}$ & 540 & 74.9043 \\
\hline 11 & $\begin{array}{l}\text { Antony and } \\
\text { Cleopatra }\end{array}$ & $\mathrm{T}$ & 18384 & 97.4471 \\
\hline 12 & King Lear & $\mathrm{T}$ & 19126 & $(73.3908)^{\star}$ \\
\hline 13 & The Tempest & $\mathrm{C}$ & 11733 & 50.0296 \\
\hline 14 & $\begin{array}{l}\text { Marry Wives of } \\
\text { Windsor }\end{array}$ & $\mathrm{C}$ & 15960 & 99.3469 \\
\hline 15 & Sonnets $1-10$ & $\mathrm{~S}$ & 884 & 97.9331 \\
\hline 16 & Sonnets $1-20$ & $\mathrm{~S}$ & 1788 & 98.3348 \\
\hline 17 & Sonnets $1-60$ & $\mathrm{~S}$ & 5250 & 98.3229 \\
\hline 18 & Sonnets $1-80$ & $\mathrm{~S}$ & 6946 & 98.3157 \\
\hline 19 & Sonnets 1-99 & $\mathrm{S}$ & 8612 & 97.8108 \\
\hline
\end{tabular}

()$^{*}=$ incorrectly attributed

The network made five incorrect attributions in the narrow categorization problem, clearly indicating a more difficult process. In addition, three other cases (samples \#5, \#10, \#13) had confidence levels below $78 \%$. Categorizing any of the 
sonnet sets is uncontroversial. The difficulty lies mostly in telling the difference between a comedy and a tragedy. Two comedy texts, excerpts from Measure for Measure and Much Ado about Nothing, are categorized as tragedies. Two tragedies, King Lear and Antony and Cleopatra are incorrectly categorized as comedies.

The results in table 4 are from a single training of the network, but are representative of about 20 similar runs. With minor exceptions, the same five incorrect attributions were made each time and the confidence levels of the correct attributions were very similar. We can thus eliminate random initial weights as a performance factor.

A closer examination reveals that sample text length has a more powerful affect on the process in narrow categorization. In the case of Antony and Cleopatra, the network incorrectly considered a 1448 word excerpt (sample \#6) as a comedy. However, an 18,384 word piece of the same play (sample \#11) was correctly identified as a tragedy. Sample \#7 which is a small section of King Lear was incorrectly attributed to comedy with high confidence. Sample \#12, which was the entire play was still attributed incorrectly but with a weaker confidence level. Text sample size appears to not be a conclusive factor in determining attribution, since some smaller pieces were attributed correctly. Larger pieces, however, are less likely to be unrepresentative of the target writing style.

This particular experiment is further complicated by Shakespeare's writing itself. Shakespearian tragedies often contain elements of genuine humor (such as court jesters) or "dark" humor which could have the same general linguistic signature as comedies. Likewise Shakespearian comedies do often contain serious subjects, violence, jealousy and disputes more often associated with tragedies.

\section{CONCLUSIONS AND FUTURE WORK}

In the realm of text attribution, we believe a distinction between "source" and "authorship" is necessary. Using our own criteria we were able to include the Bible and Linux comments as sources, even though both sets of corpora have multiple authors. Furthermore, one author (like Shakespeare or Chomsky) can have more than one style of writing which should be detectable.

Our source attribution experiments proved positive. In the broad categorization experiment we were able to show this common-word frequency technique works extremely well with our sample set. This means that word usage rate by itself is a powerful indicator of a source's style or distinctiveness. Lexical approaches such as the one we utilized have an advantage over syntactic or natural language processing techniques in that they need not access any pre-existing knowledge about language structure, form or meaning. Our attribution process can be improved even further by including other lexical criteria such as sentence-level statistics and word proximity matrices. Our neural network-centric approach is also compatible with $\mathrm{N}$-gram analysis. Common N-gram frequencies could replace common-word frequencies with little change to the actual process. Future work will examine comparison and cooperation between different lexical techniques in source attribution.

Our declining sample size problem produced surprising results. We were not expecting very small samples such as 118 words to be attributed with such high degree of confidence. Although interesting possibilities have been raised, no solid conclusion can be drawn as of this point without further experimentation. There are a number of other dependencies that future work will have to consider. We may certainly expect different results with a narrower categorization problem. In addition, we will have to run experiments with random pieces of small texts in order to help eliminate anomalies.

Although our narrow categorization network yielded some false attributions, we were able to categorize 14 of the 19 pieces successfully. One explanation may be that genre specific words, which are typically important clues, were eliminated due to our common-word-frequencies technique. One interesting experiment would be to combine a "bag of words" approach which does value unique words, with common-word-frequencies. The use of the neural net possibilities here is still promising.

Reducing the size of the output layer appears to accelerate convergence. In experiment 1 , the network converged in an average of 500 epochs while it was only 150 epochs in experiment 3. Distinctiveness of the corpora in this case was not a factor in convergence length since the Shakespearian corpora in experiment 3 proved less distinctive. More experimentation can be done with regards to the size of training corpora and number of categories.

The automated process aspect of our design was demonstrated with our software. We now have some intertwining scripts and programs that together constitute an "attribution machine." This machine depends on a dynamically defined embedded neural net to achieve attribution.

What we've demonstrated here is the digitization and analysis of one aspect of writing style with neural nets. Although, perhaps the most important aspect, word frequencies are not the only non-lexical indicators of a piece of writing. Other measures such as symbol usage, word, sentence and paragraph lengths, and word proximity usage are also available. Effectiveness of neural nets in a comprehensive source attribution solution will depend on 
how organically one can interlace difference aspects of writing into a single connectionist categorization system.

\section{ACKNOWLEDGEMENTS}

The authors would like to extend sincere thanks and gratitude to Dr. Susan Opava, Susan Rock and the Research and Graduate Programs office at the California Polytechnic State University for their help and contribution. Furthermore, we thank Cal Poly department of Computer Science and important feedback provided by professors Gene Fisher, Clark Turner and Charles Dana.

\section{REFERENCES}

[1] Engelbrecht, Andries P. Computational Intelligence: An Introduction. 2002. John Wiley and Sons. ISBN 0-470- 84870-7.

[2] Keselj, Vlado and Peng, Fuchun and Cercone, Nick and Thomas, Calvin. N-gram-based Author Profiles for Authorship Attribution. In Proceedings of the Conference Pacific Association for Computational Linguistics,
PACLING'03, Dalhousie University, Halifax, Nova Scotia, Canada, August 2003

[3] Brett Kessler, B and Nunberg, G., Schuetze H. Automatic Text Genre Detection. In Proceedings of the 35th Annual Meeting of the Association for Computational. Linguistics and the 8th Meeting of the European Chapter of the Association for Computational Linguistics, pages 32-38, Morgan Kaufmann Publishers, San Francisco CA, 1997.

[4] Negnevitsky, Michael. Artificial Intelligence. First edition 2002. Addison Wesley. ISBN 0-201-71159-1.

[5] Srinivas, B and A. Joshi. Supertagging: A Approach to Almost Parsing. Computational Linguistics, 25(2) 1999 237-265.

[6] N. Fakotakis E. Stamatatos and G. Kokkinakis. Computer-based Authorship Attribution without Lexical Measures. Computers and the Humanities, Volume 35, Issue 2, May 2001, pp. 193214

[7] N. Fakotakis E. Stamatatos and G. Kokkinakis. Automatic Authorship Attribution. In Proceedings EACL-99. 1999.

[8] N. Fakotakis E. Stamatatos and G. Kokkinakis. 2000. Automatic Text Categorization in Terms of Genre and Author. Computational Linguistics, 26(4), 471-495.

[9] The New American Standard Bible. Bible Researcher. accessed 4/2005. http://www.bible-researcher.com/nasb.html. 